\title{
Power Profiling of Embedded Analog/Mixed-Signal Systems
}

\author{
Jan Haase and Christoph Grimm \\ TU Vienna, Austria \\ haasedict.tuwien.ac.at, \\ grimmeict. tuwien.ac.at
}

\begin{abstract}
In order to optimize power consumption, it is important to know where and why power is consumed in a specific system. Power estimation gives a more or less accurate answer for the first question (where?). Knowing where power is consumed allows designers to optimize these specific components. However, the second question (why?) for the reason of power consumption, is more difficult to answer: Activities that are reason for power consumtion (e.g. addressing/routing in a WSN) are not located in a single component, but use a variety of components. However, knowing the cost of activities would pave the path to more holistic power optimization. The presentation will introduce methods for "power profiling" that assist the analysis of power consumption, assigning power consumption to both components and activities.
\end{abstract}

